\title{
Characterization of Streptococcus pluranimalium from a cattle with mastitis by whole genome sequencing and functional validation
}

Yushan Pan ${ }^{1,2^{*}+}$, Haoran An ${ }^{2,3 \dagger}$, Tong Fu' ${ }^{1}$, Shiyu Zhao ${ }^{1}$, Chengwang Zhang ${ }^{2}$, Genhui Xiao ${ }^{2}$, Jingren Zhang ${ }^{2}$, Xinfang Zhao ${ }^{1}$ and Gongzheng $\mathrm{Hu}^{1 *}$

\begin{abstract}
Background: Streptococcus pluranimalium is a new member of the Streptococcus genus isolated from multiple different animal hosts. It has been identified as a pathogen associated with subclinical mastitis, valvular endocarditis and septicaemia in animals. Moreover, this bacterium has emerged as a new pathogen for human infective endocarditis and brain abscess. However, the patho-biological properties of S. pluranimalium remain virtually unknown. The aim of this study was to determine the complete genome sequence of S. pluranimalium strain TH1 1417 isolated from a cattle with mastitis, and to characterize its antimicrobial resistance, virulence, and carbon catabolism.

Results: The genome of S. pluranimalium TH11417, determined by single-molecule real-time (SMRT) sequencing, consists of 2,065,522 base pair (bp) with a $\mathrm{G}+\mathrm{C}$ content of 38.65\%, 2,007 predicted coding sequence (CDS), 58 transfer RNA (tRNA) genes and five ribosome RNA (rRNA) operons. It contains a novel ISSp/1 element (a memeber of the IS3 family) and a $\Phi 11417.1$ prophage that carries the mef(A), msr(D) and Inu(C) genes. Consistently, our antimicrobial susceptibility test confirmed that S. pluranimalium TH11417 was resistant to erythromycin and lincomycin. However, this strain did not show virulence in murine pneumonia (intranasal inoculation, $10^{7}$ colony forming unit - CFU) and sepsis (intraperitoneal inoculation, $10^{7} \mathrm{CFU}$ ) models. Additionally, this strain is able to grow with glucose, lactose or galactose as the sole carbon source, and possesses a lactose-specific phosphoenolpyruvate-dependent phosphotransferase system (PTS).
\end{abstract}

Conclusions: We reported the first whole genome sequence of $S$. pluranimalium isolated from a cattle with mastitis. It harbors a prophage carrying the $\operatorname{mef}(\mathrm{A}), \operatorname{msr}(\mathrm{D})$ and $\ln u(\mathrm{C})$ genes, and is avirulent in the murine infection model.

Keywords: Streptococcus pluranimalium, Mastitis, Phylogenetic group, Prophage, mef(A), Inu(C), Carbon catabolism

\section{Background}

Streptococcus pluranimalium was first described as a new species of the Streptococcus genus in 1999 by Devriese et al. [1]. In sharp contrast with rather strict host restriction of many other streptococcal species, S. pluranimalium is promiscuous, in terms of its host and tissue tropism since it has been isolated from various tissues of multiple domestic animals and humans. In recent years, S. pluranimalium has been regarded as a pathogen associated with subclinical

\footnotetext{
* Correspondence: pylearn21@163.com; yaolilab@163.com

${ }^{\dagger}$ Yushan Pan and Haoran An contributed equally to this work.

${ }^{1}$ College of Animal Science and Veterinary Medicine, Henan Agricultural

University, Zhengzhou, China

Full list of author information is available at the end of the article
}

mastitis in dairy cows [1], many bovine reproductive diseases (abortion, stillbirth, vulvitis, vaginitis and metritis) [2], valvular endocarditis and septicaemia in adult broiler parents [3], septicaemia in Nile tilapia [4]. Furthermore, this bacterium has been isolated from human patients with subdural empyema, infective endocarditis, and brain abscess [5-7]. However, the biological properties and pathogenic mechanisms of S. pluranimalium are virtually unknown at the present time.

The previous S. pluranimalium isolates are oftern characterized by protein mass spectrometry and $16 \mathrm{~S}$ rRNA sequencing $[1,3,8]$. Phylogenetic relationship of this species with the other members of the Streptococcus

(C) The Author(s). 2018 Open Access This article is distributed under the terms of the Creative Commons Attribution 4.0 International License (http://creativecommons.org/licenses/by/4.0/), which permits unrestricted use, distribution, and 
genus has been established with the sequences of selected genes (e.g. 16S rRNA, rpoB, sodA, tuf, rnpB, gyrB, $d n a J$, recN, and greL) $[9,10]$. Characterization of genomic features of this new member of the Streptococcus genus contributes to better understand its resistance, virulence potential and phylogenetic relationship among Streptococci. However, the complete genome of S. pluranimalium has not been reported. The aim of this study was to sequence and analyze the whole genome of a $S$. pluranimalium isolated from a cattle with mastitis. This strain was further evaluated, in terms of its antimicrobial resistance, virulence and carbon catabolism.

\section{Methods}

\section{Strain and culture conditions}

S. pluranimalium strain TH11417 was isolated in 2015 from the milk of a cattle with mastitis in Henan province, China. The strain was cultured in Todd-Hewitt broth (Oxoid Ltd., London, UK) supplemented with $0.5 \%$ yeast extract (THY) and on tryptic soy agar (Oxoid) with 5\% $(v / v)$ sheep blood at $37^{\circ} \mathrm{C}$. The $16 \mathrm{~S}$ rRNA classification was performed according to a standard procedure using primers: Pr1 5'-AGAGTTTGATCCTGGCTCAG-3' and Pr2 5' - ACGGCTACCTTGTTACGACTT-3'.

\section{Genome sequencing and analysis}

The genomic DNA was extracted using Bacterial DNA Kit (Omega Bio-tek, Norcross, GA), according to the manufacturer's instructions. The genome sequencing of S. pluranimalium TH11417 was performed on a PacBio RSII single-molecule real-time (SMRT) sequencing instrument (Pacific Biosciences, Menlo Park, CA). The average sequencing coverage was approximately $317 x$ across the genome. The reads were assembled de novo using the hierarchical genome assembly process (HGAP) with the default settings of the SMRT Analysis v2.3.0 software package (Pacific Biosciences). The genome was annotated through the NCBI prokaryotic annotation pipeline (https://ncbi.nlm.nih.gov/).

The possible genomic islands (GIs) from TH11417 genome were predicted using IslandViewer 4 (http:// www.pathogenomics.sfu.ca/islandviewer/), and prophage components were predicted according to the PHAST (http://phast.wishartlab.com/). Genome maps of TH11417 was generated using Circos v0.64 software [11]. The comparative analysis of prophage and type VII secretion system (T7SS) was generated using EasyFig v2.2 software (http://mjsull.github.io/Easyfig/files.html).

\section{Phylogenetic analysis}

Phylogenetic tree was constructed using core genome containing 352 single-copy core genes of 68 members in the genus Streptococcus (67 Streptococci from NCBI GenBank and one in this study). The single-copy core genes were determined using the program OrthoMCL version 2.0 as described previously $[12,13]$. The orthologous protein sequences were aligned and concatenated using ClustalW version 2.0 [14]. The concatenated proteins to infer the organismal phylogeny were analyzed using approximately-maximum-likelihood algorithm in FastTree version 2.0 [15]. The mapping of S. pluranimalium was generated by iTOL v4.0.3 (http://itol.embl.de/).

\section{Antimicrobial susceptibility testing}

The antibiotic susceptibility was determined as minimal inhibitory concentration (MIC) using the broth microdilution method, following the guidelines of the Clinical and Laboratory Standards Institute [16]. The following antimicrobial agents were used: penicillin, cefotaxime, erythromycin, lincomycin, clindamycin, doxycycline, which were obtained from Sigma (Shanghai, China). S. pneumoniae ATCC 49619 was used as the quality control strain.

\section{Evaluation of the virulence of S. pluranimalium TH11417} The virulence of S. pluranimalium TH11417 was evaluated in murine pneumonia and sepsis models. Briefly, bacteria were grown to the mid-log phase and stored in $15 \%$ glycerol at $-80{ }^{\circ} \mathrm{C}$ for 2 days. Stocked bacteria were diluted in Ringer's solution (RS) to appropriate dose for infection. For pneumonia model, groups of 6 female C57BL/6 mice (6-8 weeks old, Vital River, Beijing, China) were anesthetized by avertin through intraperitoneal (i.p.) injection and inoculated with $1 \times 10^{7} \mathrm{CFU}$ bacteria in $30 \mu \mathrm{L}$ RS by intratracheal (i.t.) instillation. For sepsis model, mice were infected by i.p. with $1 \times 10^{7} \mathrm{CFU}$ bacteria in $200 \mu \mathrm{L}$ RS. Every $24 \mathrm{~h}$ post infection, blood samples $(20 \mu \mathrm{L})$ were collected from suborbital vein and plated on TSA plates with $5 \%(v / v)$ sheep blood for counting bacterial number, and the survival of mice were observed up to 7 days.

\section{Metabolism of carbohydrates}

The metabolic capacity for carbohydrates was evaluated by monitoring the growth of S. pluranimalium TH11417 in the presence of different sugars as the main carbon source. Briefly, bacterial cells were cultivated in a chemically defined medium $(\mathrm{CDM})$ as previously described [17], supplemented with $0.5 \%$ different carbon sources (glucose, lactose and galactose), respectively. Carbohydrates were purchased from Sigma (Shanghai, China). The growth phenotype was monitored by a BioTek Synergy H1 microplate reader (BioTek, Winooski, VT, USA) at $37{ }^{\circ} \mathrm{C}$ with $200 \mu$ in each well, with the optical density at $620 \mathrm{~nm}\left(\mathrm{OD}_{620}\right)$ of each sample recorded every $30 \mathrm{~min}$ up to $24 \mathrm{~h}$.

\section{Results}

The TH11417 genome is composed of 2,065,522 bp with a $\mathrm{G}+\mathrm{C}$ content of $38.65 \%$. It consists of 2,007 predicted 
CDS, 58 tRNA genes and 5 rRNA operons (Fig. 1). Five genomic islands and two prophage regions were predicted by Island Viewer and PHAST, respectively. The first prophage, designed as $\Phi 11417.1$, is $52,668 \mathrm{bp}$ in length and contains $53 \mathrm{CDS}$; the second prophage (named $\Phi 11417.2$ ) consists of 8,104 bp with 12 CDS. Phylogenetic analysis showed that $S$. hyovaginalis, $S$. thoraltensis, S. halotolerans, and S. pluranimalium form the pluranimalium group in the genus Streptococcus based on the distances calculated by approximately-maximum-likelihood algorithm (Fig. 2).

TH11417 was resistant to erythromycin $(\mathrm{MIC}=$ $16 \mu \mathrm{g} / \mathrm{mL}$ ), lincomycin ( $\mathrm{MIC}=64 \mu \mathrm{g} / \mathrm{mL})$, and susceptible to penicillin ( $\mathrm{MIC}<0.125 \mu \mathrm{g} / \mathrm{mL}$ ), cefotaxime $(\mathrm{MIC}<0.125 \mu \mathrm{g} / \mathrm{mL})$, clindamycin $(\mathrm{MIC}=0.25 \mu \mathrm{g} / \mathrm{mL})$, doxycycline $(\mathrm{MIC}=0.25 \mu \mathrm{g} / \mathrm{mL})$. The analysis of whole genome indicated that it contains the mef(A), $m s r(\mathrm{D})$ and $\ln u(C)$ genes, which confer resistance to erythromycin and lincomycin. These resistance determinants are associated with a $52.7-\mathrm{kb}$ chimeric genetic element composed of a transposon inserted into the $\Phi 11417.1$ prophage. This transposon contains the heavy metal transporter ATPase and efflux system accessory genes, $m e f(\mathrm{~A})$ and $m s r(\mathrm{D})$ resistant genes, and a mobile element ISSag10 carrying $\operatorname{lnu}(\mathrm{C})$ gene. The ISSag10 is inserted to upstream of mef(A), generating two direct repeats (DRs) (TTCTTATT) (Fig. 3a).

A new 1,430-bp insertion sequence (IS) belonging to IS3 family was identified in TH11417 and designated as ISSpl1. It is flanked by 20/25-bp imperfect inverted repeats and contains two open reading frames, which encode 178- and 304-amion-acid proteins. The whole ISSpl1 shows $81 \%$ identity to the IS 861 , which was firstly characterized in S. agalactiae COH-I [18]. Four copies of ISSpl1 were observed throughout the chromosome of TH11417, and one of the copies lacking the target sequence is located near to the genes involved in bacteriocin synthesis, other copies create 3-bp directly repeated sequences at the target site (TTC, ATT, GGG) (http://www-is.biotoul.fr/).

Analysis of the whole genome of the TH11417 revealed that it harbors several virulence-associated factors, including fibronectin-binding protein, hemolysin III homolog, cell wall anchored protein sortase and LPXTG-anchored

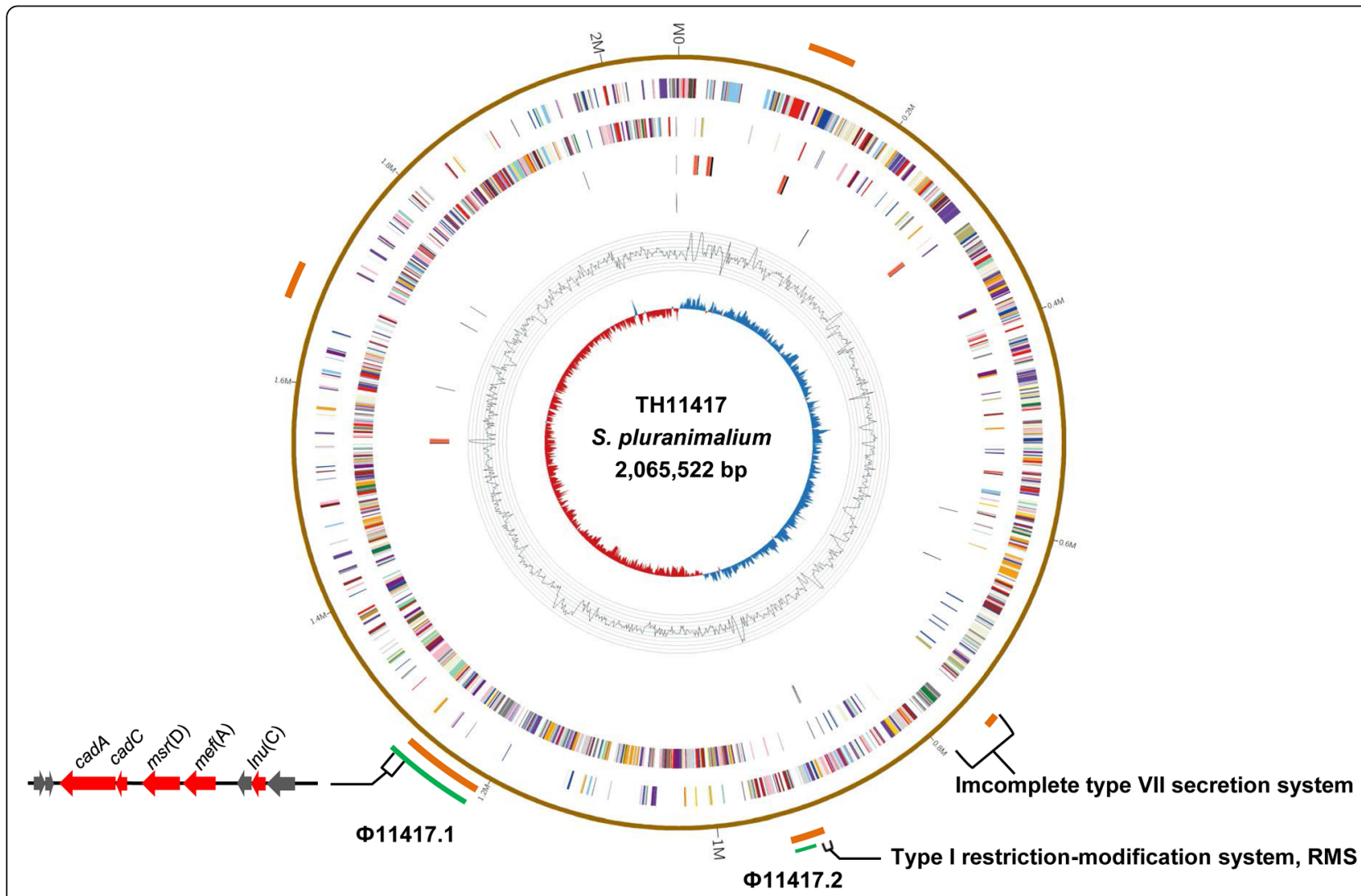

Fig. 1 Genome map of S. pluranimalium TH1 1417. Map was established using the software Circos. The circular diagrams (rings from the outermost to the center): 1) prophages predicted (green), $\Phi 11417.1$ prophage carried the $\operatorname{mef}(\mathrm{A}), \mathrm{msr}(\mathrm{D})$ and $\operatorname{lnu}(\mathrm{C})$ genes; 2) genomic island (GI) (light red); 3) scale marks of the genome; 4) protein-coding genes on the forward stand; 5) protein-coding genes on the reverse strand; 6) tRNA (black) and rRNA genes on the forward strand; 7) tRNA (black) and rRNA genes on the reverse strand; 8) GC content; 9) GC skew. Protein-coding genes are color coded according to their Cluster of Orthologous Gene Categories (COG) categories [40] 


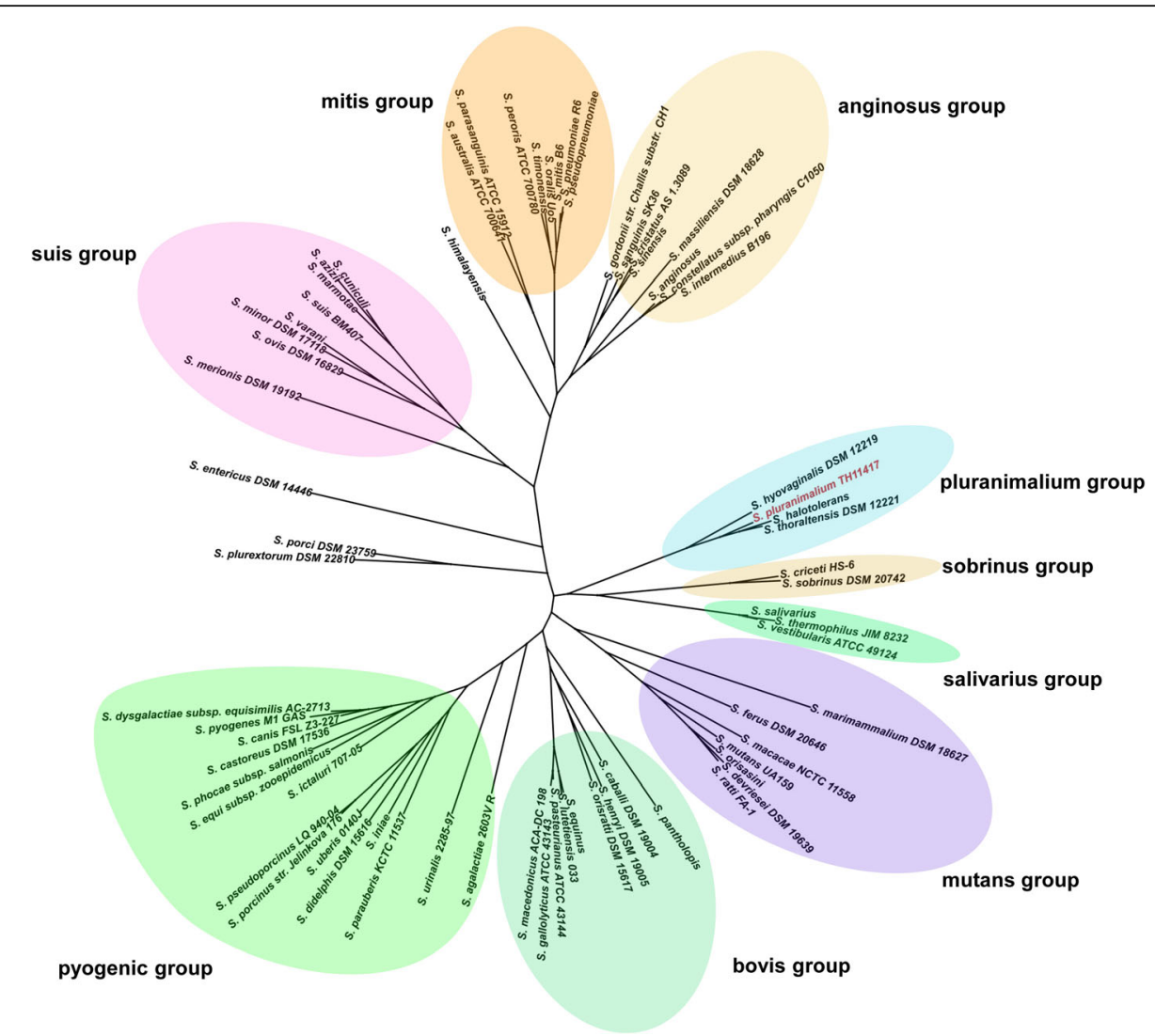

Fig. 2 Phylogenetic tree of all 68 representative genomes in the genus Streptococcus, including S. pluranimalium TH11417. The tree was reconstructed with approximately-maximum-likelihood algorithm based on 352 single-copy core genes concatenated to core genome proteins, computed by FastTree and mapping by iTOL v4.0.3 (http://itol.embl.de/)

protein, IgA1 protease. The fibronectin-binding protein and hemolysin III protein of TH11417 display high identity at protein level with the same pluranimalium group and other streptococcal species whose genomes have been published in the NCBI database, 93.3 and $92.6 \%$ with $S$. thoraltensis DSM 12221 (NZ_KB904587), 91.4.0 and 88.9\% with S. halotolerans (NZ_CP014835), 90.4 and 83.3\% with S. hyovaginalis (NZ_ATVP01000004, NZ_ ATVP01000012), respectively. BLASTp analysis showed that cell wall anchored protein sortase and LPXTG-anchored protein of TH11417 display moderate identity with that of many other streptococcal species. The IgA1 protease of TH11417 display low identity with that of many other streptococcal species. Together, the IgA1 protease in TH11417 has relatively higher specificity than other virulence-associated factors. As shown in Fig. 3b, the TH11417 genome carries a type VII secretion system (T7SS) harboring secretory antigenic target ESAT-6 (substrate protein, EsxA), secretion accessory protein EsaA and EsaB, secretion system component EssA, EssB, and Ess $C$ proteins. However, the T7SS locus is interrupted by many hypothetical genes between esx $A$ and esaA. EsxA of TH11417 was found to show $96.9 \%$ amino acid identity to the corresponding protein of S. thoraltensis DSM 12221 (NZ_KB904587), moderate identity to that of S. suis 05HAS68 (CP002007) (58.8\%) [19], and 44.3\% identity to that of Staphylococcus aureus Mu50 (BA000017) [20]. The other related secretion proteins were illustrated in detail (Fig. 3b). In addition, the several hypothetical proteins of T7SS were predicted as genomic island by Island Viewer software. So, we speculated that this T7SS is incomplete and defective.

To verify whether these putative virulence factors confer pathogenicity to S. pluranimalium, TH11417 was used to infect mice at a dose of $1 \times 10^{7} \mathrm{CFU}$ in both acute pneumonia and sepsis models that have been used to assess the virulence of S. pneumoniae [21]. No bacteria were detected in the blood of mice infected by either i.t. (pneumonia) or i.p. (sepsis) 24 to $48 \mathrm{~h}$ post infection. All of the mice survived without any obvious symptom more than 7 days post infection. This result strongly suggested that TH11417 is relatively low- or avirulence.

The ability of S. pluranimalium TH11417 to grow with glucose, lactose and galactose as the main carbohydrate source was evaluated in CDM medium supplemented with single carbohydrate. As presented in Fig. 4, 

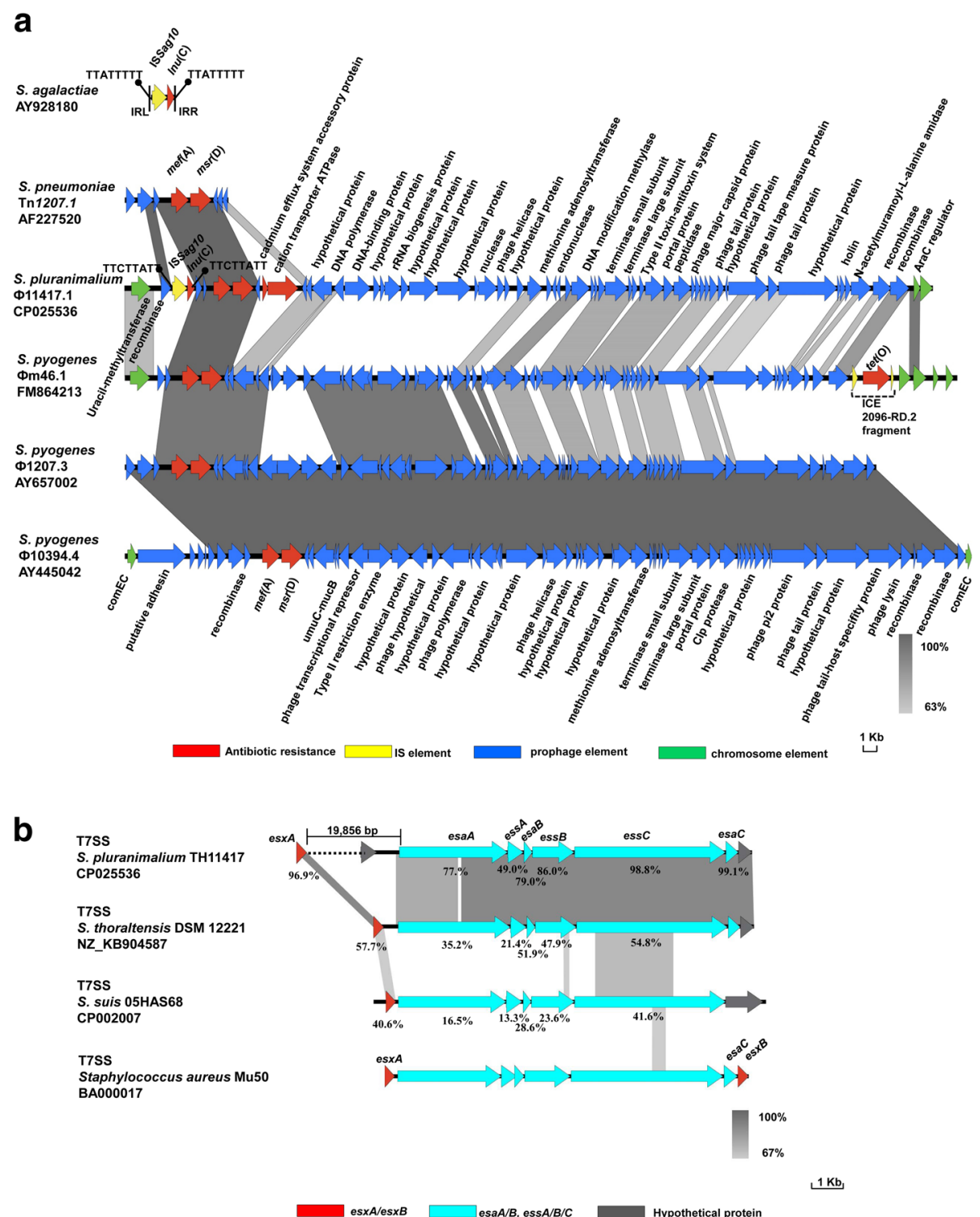

Fig. 3 Comparative analysis of the $\Phi 11417.1$ and T7SS in S. pluranimalium TH11417 with that of other strains. a Sequence comparison between the $\Phi 11417.1$ in S. pluranimalium TH1 1417 with related genetic elements and prophages, ISSag10 bearing the Inu(C) gene in S. agalactiae (AY928180), Tn1207.1 in S. pneumonia (AF227520), Ф1207.3 in S. pyogenes (AY657002), Ф10394.4 in S. pyogenes (AY445042), Фm46.1 in S. pyogenes (FM864213). The positions and orientation of transcription for resistance and other genes on each mobile genetic elements are indicated by directional arrows. Filled circles on stalks are used to indicate DRs. Homologous segments generated by a BLASTn comparison ( $\geq 63 \%$ identity) are shown as grey boxes. $\mathbf{b}$ Comparison of the S. pluranimalium TH1 1417 ESAT-6 locus with the S. thoraltensis DSM 12221 (NZ_KB9045876), S. suis 05HAS68 (CP002007) and Staphylococcus aureus Mu50 (BA000017) locus. The positions and orientation of transcription for T7SS locus are indicated by directional arrows. Homologous segments generated by a BLASTn comparison ( $\geq 67 \%$ identity) are shown as grey boxes. The identity of amino acid sequences for each gene related to the secretory antigenic targart ESAT- 6 is expressed as a percentage

TH11417 grew in the presence of glucose, lactose, or galactose. As expected, the CDM with glucose yielded the most productive growth as evidenced by the doubling time in the exponential phase $(6 \mathrm{~h})$ and maximal culture density (OD620 1.4). In contrast, the medium containing lactose or galactose showed much slower growth. Although TH11417 showed the longest lag phase in the lactose CDM but eventually showed a second highest maximal density (OD620 1.0), suggesting that lactose metabolism requires extra time for induction. Analysis of the TH11417 genome revealed that it harbors intact lactose and galactose metabolism loci (lacRABCDFEG and galRKTE). The lactose metabolism locus consists of 8 genes ( $l a c R$ and lac operon of 7 genes: lacABCDFEG). The genes in the lac locus of $S$. pluranimalium are highly similar to those of $S$. 


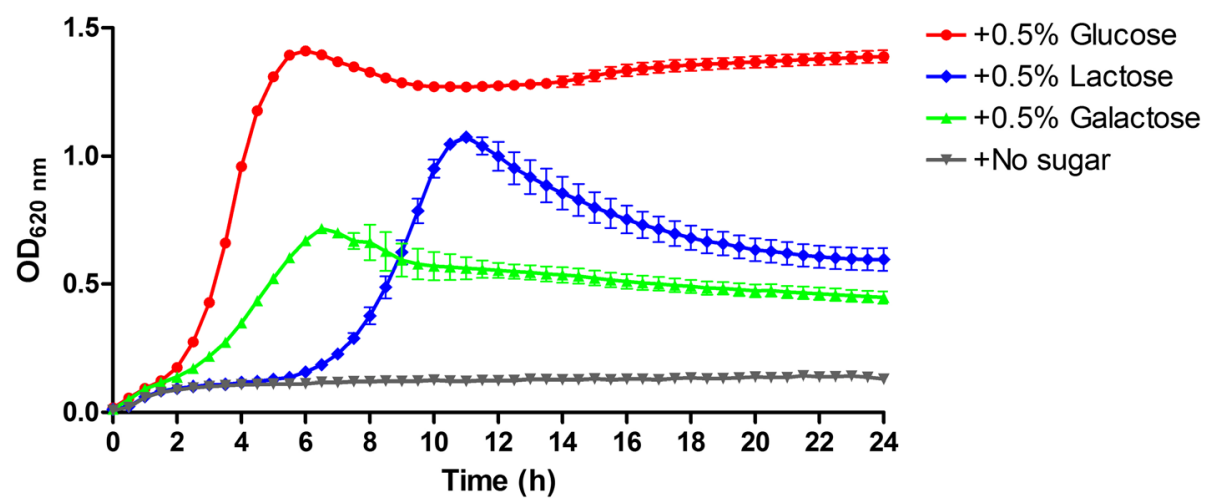

Fig. 4 Growth curves of the S. pluranimalium generated using CDM medium supplemented with $0.5 \%$ glucose, $0.5 \%$ lactose, $0.5 \%$ galactose. The values are the means \pm standard deviations

agalactiae ILRI005 in gene organization and amino acid sequence [22] (Fig. 5a). As an example, lacC, the least similar gene in the locus between the two species, has 93.2\% sequence identity. In contrast, the lac operon of $S$. pluranimalium has much lower overall sequence homology with that of S. mutans UA159 [23, 24], a well-characterized oral streptococcus (Fig. 5a). The gal operon (galRKTE) in TH11417 also has the same organization as in S. salivarius ATCC 25975 [25], however, the lactose permease lacS is absent (Fig. 5b). These results indicated that S. pluranimalium TH11417 is capable of transporting and metabolizing lactose though lactose PTS and tagatose 6-phosphate pathway.

\section{Discussion}

S. pluranimalium, was first identified by Devriese et al. In 1999 [1]. Since then, this new Streptococcus was isolated from different animals and humans. However, the complete genome of $S$. pluranimalium is still unknown. In this study, we determined the complete genome sequence of S. pluranimalium TH11417. The genus Strepococcus has been divided into nine major groups (mutans, bovis, pyogenic, suis, mitis, anginosus, pluranimalium, sobrinus, and salivarius) [10]. Phylogenetic analysis of the TH11417 genome has confirmed that $S$. pluranimalium forms the pluranimalium group with $S$. hyovaginalis, S. thoraltensis, and S. halotolerans (Fig. 2). Moreover, the S. pluranimalium genome is closely related to the streptococcal genomes in the sobrinus and salivarius groups, suggesting that pluranimalium is ancestral to these two groups (Fig. 2). Notably, S. gordonii belongs to mitis group based on analysis of the 16S rRNA gene [26], whereas S. gordonii was classified as anginosus group by single-copy core genes as well as called gordonii group using eight phylogenetic markers [10].

This study, for the first time to our best knowledge, revealed that three drug-resistance determinants mef(A), $m s r(\mathrm{D})$ and $\ln u(\mathrm{C})$ coexist in a single prophage. The
mef(A) gene encodes an efflux pump exhibiting resistance to macrolides, and susceptibility to lincosamides and streptogramin B antibiotics, which was originally described in S. pyogenes in 1996 [27]. The $m s r(D)$ gene, one of the ABC-F subfamily of ATP-binding cassette proteins, mediate macrolide resistance through ribosomal protection [28]. The $m s r(\mathrm{D})$ gene along with mef(A) was previously found on the defective transposon Tn1207.1 in S. pneumoniae, which could not be transferred by conjugation experiment [29]. However, an originally called $\operatorname{Tn} 1207.3$ conjugative transposon carrying this $m e f(\mathrm{~A}) / m s r(\mathrm{D})$ pair of genes could be transferred in different streptococcal species. Now, the Tn1207.3 was re-named as a prophage $\Phi 1207.3$ in S. pyogenes [30]. Including $\ln u(C)$ gene conferring resistance to lincomycin, several different genes have been identified and deposited in the nomenclature centre for MLS resistance genes (http://faculty.washington.edu/marilynr/), which inactivate lincosamides by adenylylation in Staphylococcus, Enterococcus, Streptococcus, Haemophilus parasuis, Riemerella anatipestifer. The ISSag10 bearing $\ln u(C)$ was first identified in S. agalactiae UCN36 in 2005, which was inserted in the operon for capsular synthesis, and generated both DRs (TTATTTTT) [31]. In the present study, the ISSag10 is simply inserted to a transposon resembling Tn1207.1 of S. pneumoniae [29] (Fig. 3a). At the sequence level, $\Phi 11417.1$ has low homology to $\Phi \mathrm{m} 46.1$, $\Phi 1207.3$ and $\Phi 10394.4$ from S. pyogenes, except for Tn1207.1-like elements [30, 32, 33]. Interestingly, the $\Phi 1207.3$ and $\Phi 10394.4$ integrate into comEC coding sequence at the same chromosomal site, whereas $\Phi 11417.1$ as well as $\Phi \mathrm{m} 46.1$ integrates into the gene encoding $23 \mathrm{~S}$ rRNA uracil methyltransferase (Fig. 3a). These results indicated that S. pluranimalium TH11417 could acquire the resistance determinants through phage horizontal transfer.

This study has identified a type VII secretion system (T7SS)-like locus in S. pluranimalium. T7SS, the newest secretion system in prokaryotic organisms, are found in 


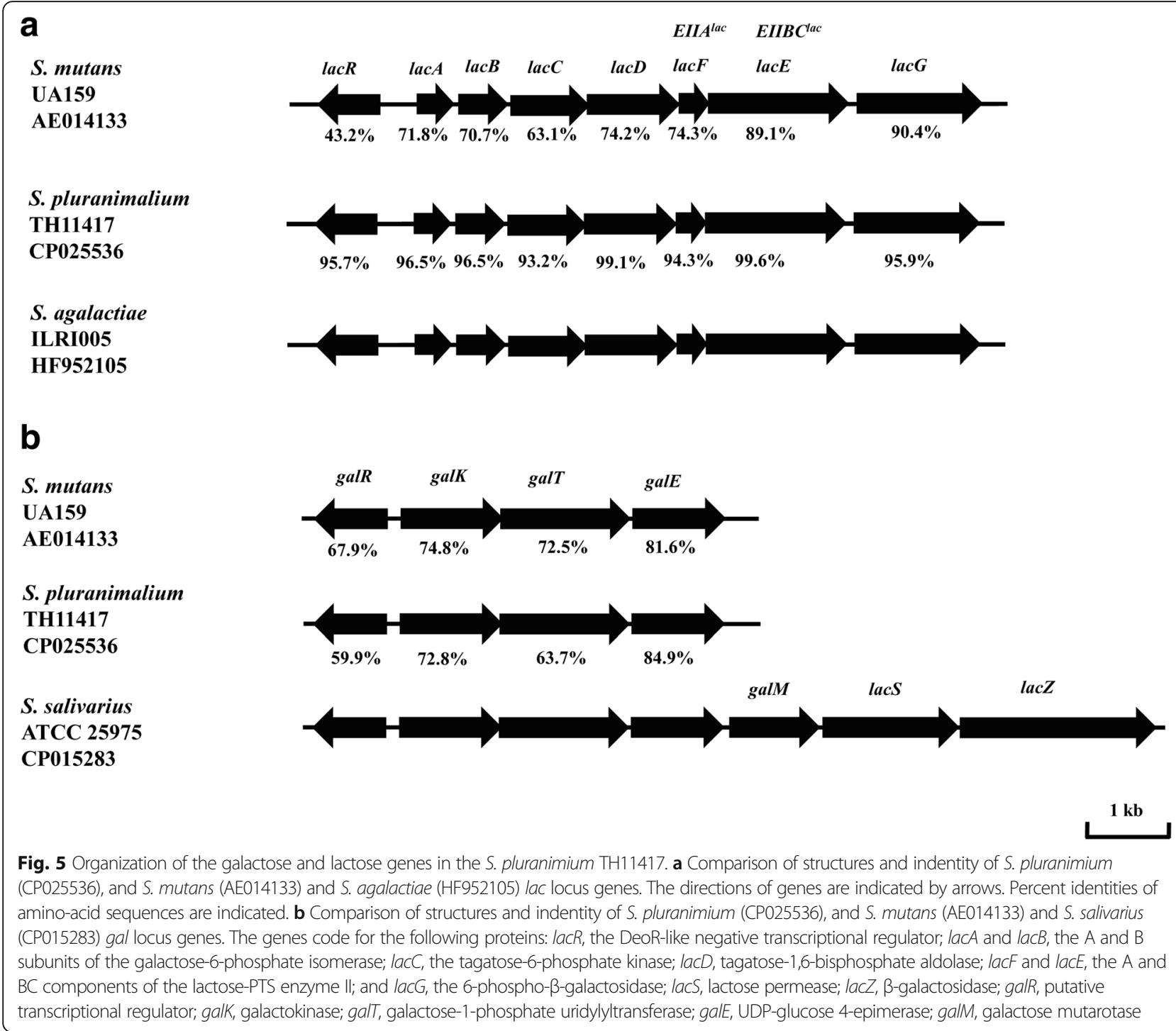

certain Gram-positive pathogens, including Mycobacteria tuberculosis and Staphylococcus aureus [34]. Very recently, Lai et al. reported a type VII secretion system in $S$. suis which contributes to virulence in a mouse infection model [35]. Although multiple virulence associated factors are found in the genome of S. pluranimalium TH11417, this strain did not show obvious virulence in both the pneumonia and sepsis mouse models. Because previous studies have shown that $S$. pluranimalium is associated with diseases in domestic animals and humans [1-6], it is possible that TH11417 is specialized in colonizing the bovine environment and lacks certain factors for successful infection in mice. The availability of the TH11417 genome will help future investigations into the genetic basis of pathogenesis and biology in this species.

Lactose is the primary carbon and energy source used by some Streptococcus strains for growth in milk [36]. In this study, we isolated S. pluranimalium TH11417 from a cattle with mastitis, which is capable of metabolizing lactose and galactose. There are multiple systems to transport/metablize a single substrate in bacteria [36, 37]. In lactose metabolism, the $\beta$-galactosidase (LacZ) is the predominant metabolic system through lactose permease (LacS) for S. salivarius 25975, while the lactose-PTS is the major metabolic pathway for $S$. mutans, both of which were induced by lactose [37]. Like bovine-adapted S. agalactiae [38], S. pluranimalium TH11417 could also metabolize lactose and galactose by two distinct pathways: tagatose 6-phosphate (lac) and Leloir (gal) passways. In S. salivarius and $S$. thermophilus, lactose is not transported by lactose-specific PTS, but solely through lactose permease (LacS), which is cleaved by $\beta$-galactosidase (LacZ). However, $S$. salivarius is able to metabolize galactose via the Leloir pathway, while $S$. thermophilus doesn't metabolize 
galactose because galK gene is poorly translated [25, 39]. In the present study, TH11417 strain harbors intact lac and gal operons, but the lactose permease lacS is absent. The genotypes are consistent with the poor growth in CDM medium with $0.5 \%$ lactose during the first $6 \mathrm{~h}$ of incubation, suggesting that the lactose-PTS is the primary metabolic pathway for lactose.

\section{Conclusions}

In conclusion, we reported the first whole genome sequence of S. pluranimalium isolated from a cattle with mastitis. The analysis of whole genome revealed that TH11417 harbors a chimeric $\Phi 11417.1$ prophage carrying Tn1207.1-like and ISSag10 transposons, and several putative virulence factors, such as a fibronectin-binding protein and a type VII secretion system-like locus. $S$. pluranimalium TH11417 transports and metabolizes lactose though lactose PTS and tagatose 6-phosphate pathway. This complete genome will be highly valuable for the genetic basis of biology and pathogenesis in this species.

\section{Abbreviations \\ CDM: Chemically defined medium; CFU: Colony forming unit; IS: Insertion sequence; MIC: Minimal inhibitory concentration; T7SS: Type VII secretion system}

\section{Acknowledgements}

Not applicable

\section{Funding}

This work was supported in part by the Science and Technology Key Project Foundation of Henan Province (No. 132102110126) and the National Natural Science Foundation of China (grant No. U1504326). The funding bodies had no role in the design of the study and collection, analysis and interpretation of data and in writing the manuscript.

\section{Availability of data and materials}

All data generated or analyzed in this study are included within the article and its additional files. The complete genome sequence of S. pluranimalium TH11417 determined in this study has been deposited in the GenBank database under accession no. CP025536. The new insert sequence ISSp/1 have been deposited in the ISfinder database (http://www-is.biotoul.fr/A.

\section{Authors' contributions}

YSP and GZH conceived and designed the experiments; HRA, TF, SYZ and XFZ carried out sample collection, processing, antimicrobial testing, and animal experiments; CWZ and GHX carried out carbon catabolism experiment; YSP, HRA and JRZ analyzed the data; YSP and HRA drafted the manuscript; JRZ revised the paper. All authors read and approved the final manuscript.

\section{Ethics approval and consent to participate}

All animal experiments were performed in accordance with the principles in the Chinese law on the humane use of animals for scientific use, and approved by the Institutional Animal Care and Use Committee in Tsinghua University with the animal protocol number 14-ZJR1.

\section{Consent for publication}

Not applicable

\section{Competing interests}

The authors declare that they have no competing interests.

\section{Publisher's Note}

Springer Nature remains neutral with regard to jurisdictional claims in published maps and institutional affiliations.

\section{Author details}

${ }^{1}$ College of Animal Science and Veterinary Medicine, Henan Agricultural University, Zhengzhou, China. ${ }^{2}$ Center for Infectious Disease Research, School of Medicine, Tsinghua University, Beijing, China. ${ }^{3}$ Tsinghua-Peking Joint Center for Life Science, School of Medicine, Tsinghua University, Beijing, China.

Received: 1 March 2018 Accepted: 29 October 2018

Published online: 12 November 2018

\section{References}

1. Devriese LA, Vandamme P, Collins MD, Alvarez N, Pot B, Hommez J, Butaye $P$, Haesebrouck F. Streptococcus pluranimalium sp. nov., from cattle and other animals. Int J Syst Bacteriol. 1999;49:1221-6.

2. Foster G, Barley J, Howie F, Falsen E, Moore E, Twomey DF, Wragg P, Whatmore AM, Stubberfield E. Streptococcus pluranimalium in bovine reproductive disease. Vet Rec. 2008;163(21):638.

3. Hedegaard L, Christensen H, Chadfield MS, Christensen JP, Bisgaard M. Association of Streptococcus pluranimalium with valvular endocarditis and septicaemia in adult broiler parents. Avian Pathol. 2009:38(2):155-60

4. Osman KM, Al-Maary KS, Mubarak AS, Dawoud TM, Moussa IMI, Ibrahim MDS, Hessain AM, Orabi A, Fawzy NM. Characterization and susceptibility of streptococci and enterococci isolated from Nile tilapia (Oreochromis niloticus) showing septicaemia in aquaculture and wild sites in Egypt. BMC Vet Res. 2017;13(1):357.

5. Aryasinghe L, Sabbar S, Kazim Y, Awan LM, Khan HK. Streptococcus pluranimalium: a novel human pathogen? Int I Surg Case Rep. 2014;5(12): 1242-6.

6. Fotoglidis A, Pagourelias E, Kyriakou P, Vassilikos V. Endocarditis caused by unusual Streptococcus species (Streptococcus pluranimalium). Hippokratia. 2015:19(2):182-5.

7. Maher G, Beniwal M, Bahubali V, Biswas S, Bevinahalli N, Siddaiah N, Srinivas D. Streptococcus pluranimalium: An emerging animal streptococcal species as a causative agent of human brain abscess. World Neurosurg. 2018;115: 208-12.

8. Matajira CE, Moreno LZ, Gomes VT, Silva AP, Mesquita RE, Doto DS, Calderaro FF, de Souza FN, Christ AP, Sato MI, et al. Evaluation of protein spectra cluster analysis for Streptococcus spp. identification from various swine clinical samples. J Vet Diagn Investig. 2017;29(2):245-9.

9. Niu L, Lu S, Hu S, Jin D, Lai X, Yang J, Chen C, Wang Y, Bai X, Lan R, et al. Streptococcus halotolerans sp. nov. isolated from the respiratory tract of Marmota himalayana in Qinghai-Tibet plateau of China. Int I Syst Evol Microbiol. 2016;66(10):4211-7.

10. Pontigo F. Moraga M, Flores SV. Molecular phylogeny and a taxonomic proposal for the genus Streptococcus. Genet Mol Res. 2015;14(3):10905-18.

11. Krzywinski M, Schein J, Birol I, Connors J, Gascoyne R, Horsman D, Jones SJ, Marra MA. Circos: an information aesthetic for comparative genomics. Genome Res. 2009;19(9):1639-45.

12. Gao XY, Zhi XY, Li HW, Klenk HP, Li WJ. Comparative genomics of the bacterial genus Streptococcus illuminates evolutionary implications of species groups. PLoS One. 2014;9(6):e101229.

13. Chen F, Mackey AJ, Stoeckert CJ Jr, Roos DS. OrthoMCL-DB: querying a comprehensive multi-species collection of ortholog groups. Nucleic Acids Res. 2006;34:363-8.

14. Larkin MA, Blackshields G, Brown NP, Chenna R, McGettigan PA, McWilliam $H$, Valentin F, Wallace IM, Wilm A, Lopez R, et al. Clustal W and Clustal X version 2.0. Bioinformatics. 2007:23(21):2947-8.

15. Price MN, Dehal PS, Arkin AP. FastTree 2 - approximately maximumlikelihood trees for large alignments. PLoS One. 2010:5(3):e9490

16. Clinical and Laboratory Standards Institute (CLSI). Performance Standards for Antimicrobial Susceptibility Testing, Twenty-Seventh Informational Supplement. Wayne, PA. 2017;M100-S27:78-83.

17. van de Rijn I, Kessler RE. Growth characteristics of group a streptococci in a new chemically defined medium. Infect Immun. 1980;27(2):444-8.

18. Rubens CE, Heggen LM, Kuypers JM. IS861, a group B streptococcal insertion sequence related to IS150 and IS3 of Escherichia coli. J Bacteriol. 1989;171(10):5531-5. 
19. Yao X, Li M, Wang J, Wang C, Hu D, Zheng F, Pan X, Tan Y, Zhao Y, Hu L, et al. Isolation and characterization of a native avirulent strain of Streptococcus suis serotype 2: a perspective for vaccine development. Sci Rep. 2015;5:9835.

20. Burts ML, Williams WA, DeBord K, Missiakas DM. EsxA and EsxB are secreted by an ESAT-6-like system that is required for the pathogenesis of Staphylococcus aureus infections. Proc Natl Acad Sci U S A. 2005;102(4):1169-74.

21. Wen Z, Sertil O, Cheng Y, Zhang S, Liu X, Wang WC, Zhang JR. Sequence elements upstream of the core promoter are necessary for full transcription of the capsule gene operon in Streptococcus pneumoniae strain D39. Infect Immun. 2015;83(5):1957-72

22. Zubair S, de Villiers EP, Younan M, Andersson G, Tettelin H, Riley DR, Jores J, Bongcam-Rudloff E, Bishop RP. Genome Sequences of Two Pathogenic Streptococcus agalactiae Isolates from the One-Humped Camel Camelus dromedarius. Genome Announc. 2013;1 (4):e00515-13.

23. Zeng L, Das S, Burne RA. Utilization of lactose and galactose by Streptococcus mutans: transport, toxicity, and carbon catabolite repression. J Bacteriol. 2010;192(9):2434-44.

24. Abranches J, Chen YY, Burne RA. Galactose metabolism by Streptococcus mutans. Appl Environ Microbiol. 2004;70(10):6047-52.

25. Vaillancourt K, Moineau S, Frenette M, Lessard C, Vadeboncoeur C. Galactose and lactose genes from the galactose-positive bacterium Streptococcus salivarius and the phylogenetically related galactose-negative bacterium Streptococcus thermophilus: organization, sequence, transcription, and activity of the gal gene products. J Bacteriol. 2002;184(3):785-93.

26. Kawamura Y, Hou XG, Sultana F, Miura H, Ezaki T. Determination of 165 rRNA sequences of Streptococcus mitis and Streptococcus gordonii and phylogenetic relationships among members of the genus Streptococcus. Int J Syst Bacteriol. 1995;45(2):406-8.

27. Clancy J, Petitpas J, Dib-Hajj F, Yuan W, Cronan M, Kamath AV, Bergeron J, Retsema JA. Molecular cloning and functional analysis of a novel macrolideresistance determinant, mef(a), from Streptococcus pyogenes. Mol Microbiol. 1996;22(5):867-79

28. Sharkey LK, Edwards TA, O'Neill AJ. ABC-F proteins mediate antibiotic resistance through ribosomal protection. MBio. 2016;7(2):e01975.

29. Santagati M, lannelli F, Oggioni MR, Stefani S, Pozzi G. Characterization of a genetic element carrying the macrolide efflux gene mef(a) in Streptococcus pneumoniae. Antimicrob Agents Chemother. 2000;44(9):2585-7.

30. lannelli F, Santagati M, Santoro F, Oggioni MR, Stefani S, Pozzi G. Nucleotide sequence of conjugative prophage $\$ 1207.3$ (formerly Tn1207.3) carrying the $\mathrm{mef}(\mathrm{A}) / \mathrm{msr}(\mathrm{D})$ genes for e ffl ux resistance to macrolides in Streptococcus pyogenes. Front Microbiol. 2014;5:687.

31. Achard A, Villers C, Pichereau V, Leclerca R. New Inu(C) gene conferring resistance to lincomycin by nucleotidylation in Streptococcus agalactiae UCN36. Antimicrob Agents Chemother. 2005;49(7):2716-9.

32. Banks DJ, Porcella SF, Barbian KD, Martin JM, Musser JM. Structure and distribution of an unusual chimeric genetic element encoding macrolide resistance in phylogenetically diverse clones of group a Streptococcus. J Infect Dis. 2003;188(12):1898-908.

33. Brenciani A, Bacciaglia A, Vignaroli C, Pugnaloni A, Varaldo PE, Giovanetti E. Фm46.1, the main Streptococcus pyogenes element carrying mef(a) and tet(O) genes. Antimicrob Agents Chemother. 2010;54(1):221-9.

34. Bottai D, Groschel MI, Brosch R. Type VII secretion Systems in Gram-Positive Bacteria. Curr Top Microbiol Immunol. 2017:404:235-65.

35. Lai L, Dai J, Tang H, Zhang S, Wu C, Qiu W, Lu C, Yao H, Fan H, Wu Z. Streptococcus suis serotype 9 strain GZ0565 contains a type VII secretion system putative substrate EsxA that contributes to bacterial virulence and a vanZ-like gene that confers resistance to teicoplanin and dalbavancin in Streptococcus agalactiae. Vet Microbiol. 2017;205:26-33.

36. de Vos WM, Vaughan EE. Genetics of lactose utilization in lactic acid bacteria. FEMS Microbiol Rev. 1994;15(2-3):217-37.

37. Hamilton IR, Lo GC. Co-induction of beta-galactosidase and the lactose-Penolpyruvate phosphotransferase system in Streptococcus salivarius and Streptococcus mutans. J Bacteriol. 1978;136(3):900-8.

38. Richards VP, Choi SC, Pavinski Bitar PD, Gurjar AA, Stanhope MJ. Transcriptomic and genomic evidence for Streptococcus agalactiae adaptation to the bovine environment. BMC Genomics. 2013;14:920

39. Chen YY, Betzenhauser MJ, Snyder JA, Burne RA. Pathways for lactose/galactose catabolism by Streptococcus salivarius. FEMS Microbiol Lett. 2002;209(1):75-9.

40. Tatusov RL, Fedorova ND, Jackson JD, Jacobs AR, Kiryutin B, Koonin EV, Krylov DM, Mazumder R, Mekhedov SL, Nikolskaya AN, et al. The COG database: an updated version includes eukaryotes. BMC Bioinf. 2003;4:41.

\section{Ready to submit your research? Choose BMC and benefit from}

- fast, convenient online submission

- thorough peer review by experienced researchers in your field

- rapid publication on acceptance

- support for research data, including large and complex data types

- gold Open Access which fosters wider collaboration and increased citations

- maximum visibility for your research: over $100 \mathrm{M}$ website views per year

At BMC, research is always in progress.

Learn more biomedcentral.com/submissions 